\title{
Serial Weekly Measurements of the Timed Inspiratory Effort Index Can Predict Successful Prolonged Weaning
}

\author{
Bruno Leonardo da Silva Guimarães, Leonardo Cordeiro de Souza, Fernando Silva Guimarães, and \\ Jocemir Ronaldo Lugon
}

\begin{abstract}
BACKGROUND: Patients who require prolonged weaning from mechanical ventilation represent a major challenge in intensive care, and the timed inspiratory effort (TIE) index has been shown to be a clinically valuable tool to predict weaning success. OBJECTIVE: To evaluate whether weekly serial measurements of the TIE index can predict the success in prolonged weaning. METHODS: A prospective observational study in which the subjects who started the weaning process off mechanical ventilation underwent weekly measurements of the TIE index. The area under the receiver operating characteristic curve was used to evaluate the accuracy of the TIE index as a predictor of weaning success. A multivariate Cox regression model was developed to test the association of TIE index values of $\geq 1.0 \mathrm{~cm} \mathrm{H} \mathrm{H}_{2} \mathrm{O} / \mathrm{s}$ with a failure of weaning. RESULTS: Seventy subjects were selected. Their median (IQR) age was $72(62-78) \mathrm{y}$, the median (IQR) duration of mechanical ventilation was 17.5 (14-28) d, and the median (IQR) APACHE II (Acute Physiology and Chronic Health Evaluation) II score was 28 (24-31). Thirty-five of the 70 subjects (50\%) died, 22 of them after successful weaning. A total of 224 tests were performed over $56 \mathrm{~d}$. The area under the receiver operating characteristic curve of the TIE index was 0.93 . In the analysis of the probability of success during the follow-up (Kaplan-Meier method), a significant difference was obtained in favor of those with a TIE index of $\geq 1.0 \mathrm{~cm} \mathrm{H}_{2} \mathrm{O} / \mathrm{s}(53 \%$ vs $32 \%, P=.030)$. In the multivariate Cox regression analysis, values of the TIE index $\geq 1.0 \mathrm{~cm} \mathrm{H} \mathrm{H}_{2} \mathrm{O} / \mathrm{s}$ revealed an inverse, strong, and independent association with failure (hazard ratio $0.36,95 \% \mathrm{CI} 0.15-0.91 ; P=.030$ ). The following variables were also found to have an independent but direct association with failure: age and length of time before weaning. CONCLUSIONS: When measured weekly, a TIE index of $\geq 1.0 \mathrm{~cm} \mathrm{H} \mathrm{H}_{2} \mathrm{O} / \mathrm{s}$ was a good predictor of success in subjects who required prolonged weaning in our hospital. Key words: mechanical ventilation; prolonged weaning; respiratory muscles. [Respir Care 2019;64(10):1286-1292. (C) 2019 Daedalus Enterprises]
\end{abstract}

\section{Introduction}

Weaning is a critical step in the process of transition from mechanical ventilation to spontaneous breathing. ${ }^{1,2}$

\footnotetext{
Mr da Silva Guimarães is affiliated with the Medical Science Postgraduation Program, Universidade Federal Fluminense, Niteroi, Brazil. Dr de Souza is affiliated with the Physical Therapy School, Universidade Estácio de Sá, Physical Therapy Service of Hospital Icaraí and Hospital e Clínica São Gonçalo, Niteroi, Brazil. Dr Guimarães is affiliated with the Physical Therapy School, Universidade Federal do Rio de Janeiro, Rio de Janeiro, Brazil. Dr Lugon is affiliated with the Nephrology Division, Department of Medicine, Universidade Federal Fluminense, Niteroi, Brazil.

This study was partially supported by Universidade Estácio de Sá by a research productivity program.
}

Mechanical ventilation is associated with ventilatorinduced diaphragmatic dysfunction, which may start within $18 \mathrm{~h}$ of controlled ventilation, ${ }^{3}$ which leads to respiratory muscle weakness and the inability to sustain spontaneous breathing. ${ }^{4-7}$ The most recent weaning classification is based on the duration of ventilation after the first weaning attempt. ${ }^{8,9}$ Therefore, prolonged weaning is defined

\footnotetext{
The authors have disclosed no conflicts of interest.

Correspondence: Leonardo Cordeiro de Souza PhD PT, Physical Therapy Service of Hospital Icaraí and Hospital e Clínica São Gonçalo, Rua Marquês de Paraná, 233 - 5o. andar., CEP 24030-215 - Centro, Niterói, Brazil. E-mail: leonardo.uti@gmail.com.
}

DOI: $10.4187 /$ respcare.06367 
as the dependence on mechanical ventilation for $>7 \mathrm{~d}$ after the onset of spontaneous breathing trials. It is estimated that $\sim 15 \%$ of patients with a difficult weaning progress to prolonged ventilation, $5,6,8$ which results in an increased hospital length of stay, high mortality rate (47\%), and elevated costs. ${ }^{4,10-12}$ Interventions to restore muscle strength and ventilatory independence would be of great value in this setting. In addition, diagnostic tools to evaluate respiratory muscle function could be helpful to guide starting the weaning process. ${ }^{13}$

Indexes to predict weaning outcomes have been used worldwide for decades, especially in difficult cases. ${ }^{6}$ Two indexes are among the most used and recommended by the sixth American Thoracic Society and European Respiratory Society consensus 8 : the relationship between breathing frequency and tidal volume (rapid shallow breathing index), and maximum inspiratory pressure $\left(\mathrm{P}_{\text {Imax }}\right)$. In this regard, a recently proposed weaning index, the timed inspiratory effort (TIE) index exhibited a better performance than previously reported indexes. ${ }^{6,13-15}$ The timed inspiratory effort (TIE) index is calculated as the ratio of the $\mathrm{P}_{\text {Imax }}$ registered after the first $30 \mathrm{~s}$ of observation to the corresponding time required to reach this value with airway occlusion for up to 60 s. ${ }^{15}$ Based on the aforementioned information, we think it is timely to search for tools capable of early identification of the optimum time to initiate the weaning process with a high probability of success. The objective of the present study was to evaluate whether weekly serial measures of the TIE index can predict the success of prolonged weaning off mechanical ventilation in subjects with a tracheostomy.

\section{Methods}

This was a prospective observational cohort study conducted at the ICU of the Hospital Naval Marcílio Dias, Rio de Janeiro, Brazil, from December 2015 to July 2017. Subjects who met eligibility criteria had the TIE index recorded weekly to evaluate its predictive power related to weaning outcome. Subjects were enrolled in the study if they were ages $>18 \mathrm{y}$, had a tracheostomy and a weaning time of $>7 \mathrm{~d}$ after the first weaning attempt, resolution of the acute phase of the disease that led to mechanical ventilation, adequate cough reflex, and no excessive tracheobronchial secretion. Also, subjects who had any infection under control, stable cardiovascular status (heart rate $\leq 120$ beats/min and systolic blood pressure between 90 and $160 \mathrm{~mm} \mathrm{Hg}$, no or minimum use of vasopressors), stable metabolic status, hemoglobin $>10 \mathrm{~g} / \mathrm{dL}$, adequate oxygenation (arterial oxygen saturation of $>90 \%$ with $\mathrm{F}_{\mathrm{IO}_{2}} \leq 0.4$ or the ratio of oxygen blood pressure by inspiratory fraction of oxygen $(\mathrm{P} / \mathrm{F}$ ratio $) \geq 150$, with a final positive expiratory pressure of $\leq 8 \mathrm{~cm} \mathrm{H}_{2} \mathrm{O}$, adequate breathing frequency

\section{QUICK LOOK}

\section{Current knowledge}

Patients requiring prolonged weaning have a high mortality rate. A recently reported weaning predictor, the timed inspiratory effort (TIE) index, combines the ability to generate an inspiratory pressure with the time needed to achieve the maximum inspiratory pressure.

\section{What this paper contributes to our knowledge}

In a specific group of subjects with a low level of consciousness and difficult weaning, the TIE index demonstrated good ability to predict weaning outcome. Age and length of time before weaning were found to have a direct association with weaning failure.

( $\leq 35$ breaths/min), supportive pressure of $\leq 20 \mathrm{~cm} \mathrm{H}_{2} \mathrm{O}$, no significant respiratory acidosis $(\mathrm{pH}>7.30)$, and a Richmond Agitation-Sedation Scale (RASS) score of $\leq 0 .{ }^{16}$

We excluded patients older than $86 \mathrm{y}$, with tracheal stenosis, intracranial pressure $>20 \mathrm{~mm} \mathrm{Hg}$, on sedation, with severe cardiac insufficiency or hemodynamic instability, with signs of systemic infection and/or reinfection during the weaning process, and discontinuation due to intercurrences. When adopting the RASS, whose score ranges from -5 to 4 , patients who scored $>1$ were excluded. The consciousness levels of the included cases were categorized as alert and non-alert in which alertness was defined by objective actions such as opening eyes in response to a voice, following simple commands, and/or having a RASS score of -1 to +1 . Weaning was considered successful if spontaneous breathing was sustained for $\geq 48 \mathrm{~h}$ after withdrawal from mechanical ventilation. ${ }^{6-9,14,17}$ This study was approved by the research ethics committee of the Hospital Naval Marcílio Dias - Marinha does Brasil (CAAE 50264415.2.000.5256). Informed consent was obtained from every subject whenever possible or from his or her next of kin.

\section{Procedures}

All study subjects were on mechanical ventilation with the ventilator model PB840 (Covidien-Nellcor and Puritan Bennett, Boulder, Colorado) and continuously monitored by using the multi-parameter DX 2010 monitor (Dixtal, São Paulo, Brazil). After categorization as prolonged weaning and tracheostomized, subjects on pressure-support ventilation who were able to start the weaning process were 


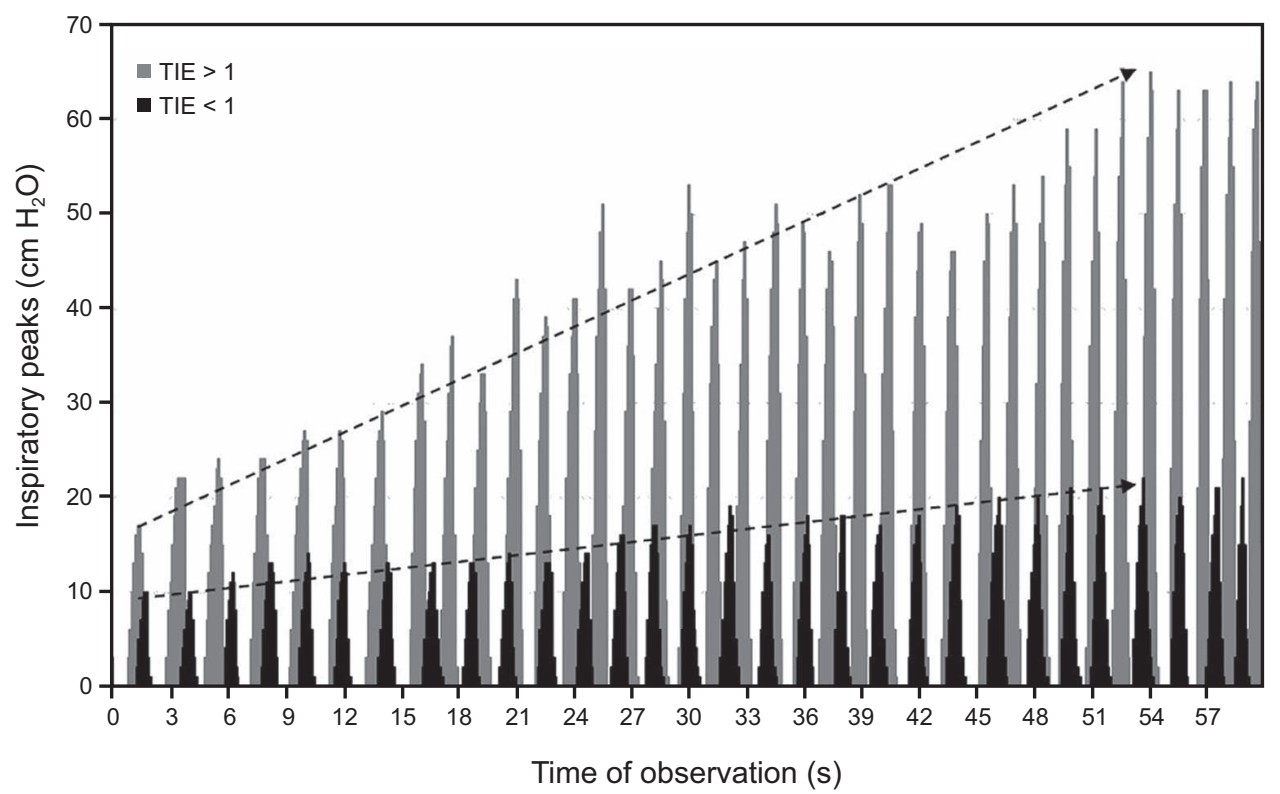

Fig. 1. Typical plots of the inspiratory pressure vs time of observation, variables used to calculade the timed inspiratory effort (TIE) index. In gray: $\mathrm{TIE}=65 \mathrm{~cm} \mathrm{H}_{2} \mathrm{O} / 54 \mathrm{~s}=1.20 \mathrm{~cm} \mathrm{H}_{2} \mathrm{O} / \mathrm{s}$. In black: $\mathrm{TIE}=22 \mathrm{~cm} \mathrm{H} \mathrm{O}_{2} \mathrm{O} / 54 \mathrm{~s}=0.41 \mathrm{~cm} \mathrm{H}_{2} \mathrm{O} / \mathrm{s}$.

followed up weekly by using the timed inspiratory effort index, as measured by the physiotherapist (BLSG). Regardless of the value obtained, all the subjects were placed on our mechanical ventilation discontinuation protocol by using respiratory training with the T-piece attached to low oxygen flow (3-5 L/min). The subjects had daily T-piece trials, with progressing spontaneous breathing time according to the protocol, then were returned to mechanical ventilation.

Criteria to interrupt periods of spontaneous breathing in T-piece trial were the following: agitation and/or anxiety, reduced level of consciousness, diaphoresis, dyspnea and/or cyanosis, $\mathrm{S}_{\mathrm{aO}_{2}}<90 \%$ or $\mathrm{P}_{\mathrm{aO}_{2}}<60 \mathrm{~mm} \mathrm{Hg}$, with $\mathrm{F}_{\mathrm{IO}_{2}}>0.4$, $\mathrm{P}_{\mathrm{aCO}_{2}}>50 \mathrm{~mm} \mathrm{Hg}$ or increased $>8 \mathrm{~mm} \mathrm{Hg}$; arterial $\mathrm{pH}$ of $<7.33$ or decreased by $\geq 0.07$, frequency of $>35$ breaths/ min or increased by $50 \%$ for $5 \mathrm{~min}$ or longer, heart rate of $>140$ beats/min or a sustained increase or decrease of $>20 \%$, mean blood pressure of $>130$ or $<70 \mathrm{~mm} \mathrm{Hg}$, and tachyarrhythmias. ${ }^{6-9,14,17}$ The subjects who demonstrated one or more of these signs during the spontaneous breathing test or within $48 \mathrm{~h}$ after discontinuation of mechanical ventilation were labeled as weaning failure and were returned to ventilatory support. ${ }^{6-9,14,18}$ The decision to return the subject to mechanical ventilation was made by a respiratory physiotherapist (BLSG) and the physician in charge based on the signs of poor tolerance, as described above.

The timed inspiratory effort index and $\mathrm{P}_{\mathrm{Imax}}$ were recorded by using the digital vacuometer MVD 300 (Globalmed, Porto Alegre, Brazil), with a scale of $300 \mathrm{~cm} \mathrm{H}_{2} \mathrm{O}$, sensitivity of $1 \mathrm{~cm} \mathrm{H}_{2} \mathrm{O}$, and a sample frequency of $100 \mathrm{~ms}^{14}$
Before testing, all the subjects were on ventilation by using the pressure-support mode. The subjects were positioned in dorsal decubitus, with the head elevated at $45^{\circ}$, and the tracheostomy cuff was inflated to avoid leakage during the measurement. After tracheal aspiration, the subjects remained connected to the mechanical ventilator with a $1.0 \mathrm{~F}_{\mathrm{IO}_{2}}$ for $2 \mathrm{~min}$, aimed at preventing hypoxemia during the measurements. ${ }^{14}$ The mechanical ventilator was disconnected, and a 10-s spontaneous breathing period ensued (to allow adaptation to the breathing mode without positive pressure).

The vacuometer was coupled to the tracheostomy cannula at the end of a normal expiration (at the level of functional residual capacity). The inspiratory pressures, in $\mathrm{cm} \mathrm{H}_{2} \mathrm{O}$, and their corresponding registration time points, in seconds, were stored and analyzed. Typical plots of the inspiratory pressures versus time with the timed inspiratory effort index of $>1$ (successful weaning) and of $<1$ (failed weaning) is depicted in Figure 1). The timed inspiratory effort index was calculated as the ratio of the $\mathrm{P}_{\mathrm{Imax}}$ registered after the first $30 \mathrm{~s}$ of observation by the corresponding time to reach during inspiration, whereas keeping the airway occluded during inspiration with a unidirectional valve for up to 60 s. ${ }^{15}$ The subjects were not coached for the maneuver, which allowed simultaneous determination of the timed inspiratory effort index and $\mathrm{P}_{\text {Imax }}$. For each subject, the first timed inspiratory effort index was measured at the beginning of the prolonged weaning process and the last was measured the week before successful weaning or death. 


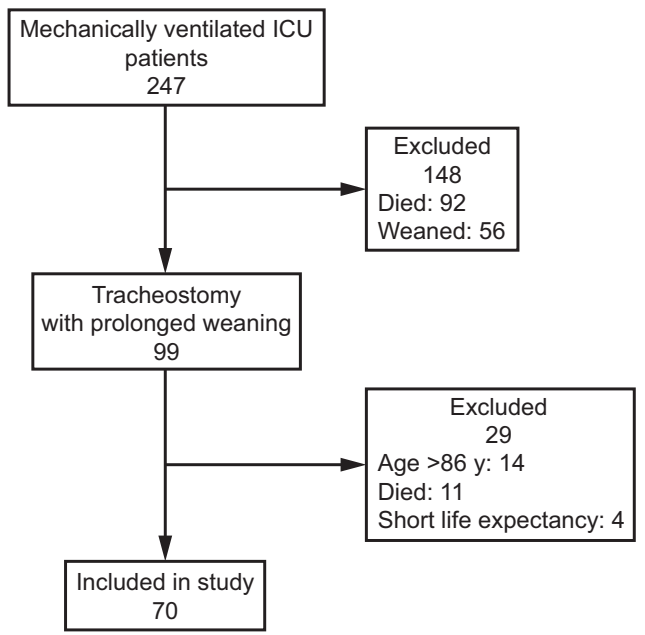

Fig. 2. Flow chart.

\section{Statistical Analysis}

For descriptive statistics, median (IQR), and frequency were used. The following indicators were used to record the performance of the timed inspiratory effort index in predicting the weaning outcome: sensitivity, specificity, positive predictive value, negative predictive value, positive likelihood ratio, and negative likelihood ratio. The accuracy of the timed inspiratory effort index was also assessed by calculating the area under the receiver operating characteristic curve. The cutoff points were calculated by using the Youden index. The outcomes along the follow-up were shown as Kaplan Meier curves factored by the timed inspiratory effort index and compared by using the log-rank test.

A multivariate Cox proportional hazards regression model was used to test the association with failure at prolonged weaning in which the primary interest variable was the timed inspiratory effort index as a dichotomic variable (values $\geq 1.0$ ). Other variables included for their potential to influence the outcome were age, sex, APACHE II (Acute Physiology and Chronic Health Evaluation II) score, length to start weaning, weaning duration, number of interruptions of weaning trials, and alertness. The level of significance was set at $5 \%$. All statistical analyses were performed by using MedCalc 11.4.2.0 (MedCalc Software, Mariakerke, Belgium) and SPSS 18.0 for Windows (SPSS, Chicago, Illinois).

\section{Results}

From a total of 247 patients on mechanical ventilation in the ICU during the study period, 70 subjects were selected for the final analysis. Details about the selection process are depicted in Figure 2. The characteristics of the 70 subjects included in the study are presented in Table 1 .
Table 1. General Characteristics of the Subjects

\begin{tabular}{lc}
\hline \hline \multicolumn{1}{c}{ Parameter } & Value \\
\hline Age, median (IQR y) & $72(62-78)$ \\
Male/female, $n(\%)$ & $35(50) / 35(50)$ \\
White/nonwhite, $n(\%)$ & $30(43) / 40(57)$ \\
Death in ICU, $n(\%)$ & $35(50)$ \\
APACHE II score, median (IQR) & $28(24-31)$ \\
Time on mechanical ventilation, median (IQR) d & $17.5(14-28)$ \\
Conditions that precipitated ICU admission, $n(\%)$ & \\
Sepsis & $38(55)$ \\
Stroke & $11(16)$ \\
Abdominal surgery & $5(7)$ \\
Brain trauma & $5(7)$ \\
COPD & $3(4)$ \\
Cardiac arrest & $3(4)$ \\
ARDS & $2(3)$ \\
Miscellaneous & $3(4)$ \\
& \\
\hline$N=70$. & \\
IQR $=$ internal quartile range \\
APACHE II = Acute Physiology and Chronic Health Evaluation II \\
\end{tabular}

A total of 224 tests were performed over $56 \mathrm{~d}$. In 4 subjects $(6 \%)$, all with a diagnosis of sepsis, the tests were interrupted before the standardized $60 \mathrm{~s}$ of airway occlusion. All of these subjects were promptly reconnected to the ventilator and recovered without any consequence.

The timed inspiratory effort index was measured weekly, from the beginning of the protocol until the end of the weaning process. Forty-nine subjects were successfully weaned. In the subjects for whom weaning failed, the baseline and final median (IQR) TIE index values were similar, $0.67(0.44-0.81) \mathrm{cm} \mathrm{H}_{2} \mathrm{O} / \mathrm{s}$ versus $0.65(0.42-0.77) \mathrm{cm}$ $\mathrm{H}_{2} \mathrm{O} / \mathrm{s}, P=.20$. In those who were successfully weaned, a statistically significant increase was found in the median (IQR) TIE index, $0.82(0.65-0.97) \mathrm{cm} \mathrm{H}_{2} \mathrm{O} / \mathrm{s}$ to 1.55 (1.29$2.15) \mathrm{cm} \mathrm{H}_{2} \mathrm{O} / \mathrm{s},(P<.001)$. A median (IQR) of $3.2(2-6)$ timed inspiratory effort index measurements were taken before a value of $\geq 1.0$ was achieved in the subjects who were successfully weaned. The mean time to achieve the $\mathrm{P}_{\text {Imax }}$ was $45.02 \pm 11.88 \mathrm{~s}$.

The cutoff points and the results of the TIE index performance (sensitivity, specificity, positive predictive value, negative predictive value, positive likelihood ratio, and negative likelihood ratio) are presented in Table 2. The area under the receiver operating characteristic curve was $0.93, P=.001$ (Fig. 3). In the analysis of the probability of success during the follow-up time (Kaplan-Meier curve), a significant difference was obtained in favor of those with a timed inspiratory effort index of $\geq 1.0 \mathrm{~cm} \mathrm{H}_{2} \mathrm{O} / \mathrm{s}$ (53\% vs $32 \%, P=.030$ ) (Fig. 4).

In the multivariate Cox regression model to test the association with failure at prolonged weaning (Table 3), the TIE index of $\geq 1.0 \mathrm{~cm} \mathrm{H}_{2} \mathrm{O} / \mathrm{s}$ revealed an inverse and 
Timed Inspiratory Effort Index to Predict Weaning

Table 2. Quality Indicators of the Timed Inspiratory Effort Index as a Predictor of Successful Weaning

\begin{tabular}{|c|c|c|c|c|c|c|}
\hline Parameter & $\begin{array}{l}\text { Sensitivity } \\
(\%)\end{array}$ & $\begin{array}{l}\text { Specificity } \\
(\%)\end{array}$ & $\begin{array}{c}\text { Positive } \\
\text { Likelihood Ratio }\end{array}$ & $\begin{array}{c}\text { Negative } \\
\text { Likelihood Ratio }\end{array}$ & $\begin{array}{c}\text { Positive Predictive } \\
\text { Value }(\%)\end{array}$ & $\begin{array}{c}\text { Negative Predictive } \\
\text { Value }(\%)\end{array}$ \\
\hline TIE index $\geq 1.0 \mathrm{~cm} \mathrm{H}_{2} \mathrm{O} / \mathrm{s}^{*}$ & 86.4 & 96.2 & 22.45 & 0.14 & 97.4 & 80.6 \\
\hline
\end{tabular}

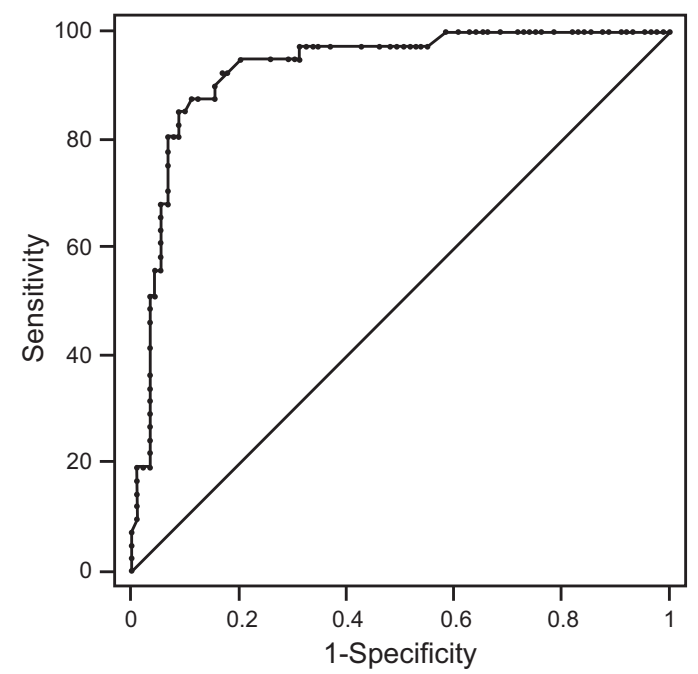

Fig. 3. The area under the receiver operating characteristic curve, evaluating the timed inspiratory effort index as a predictor of weaning success. Area under the curve of $0.93, P=.001$.

independent association with the outcome (hazard ratio $0.36,95 \%$ CI $0.15-0.91 ; P=.030$ ). Age (hazard ratio $1.04,95 \%$ CI $1.01-1.07 ; P=.03$ ) and the length of time to start the weaning process (hazard ratio $1.03,95 \% \mathrm{CI}$ $1.01-1.05 ; P=.037$ ) also showed an independent association with failure. A trend was also observed toward an association between the number of weaning trial interruptions and outcome, but this was not statistically significant (hazard ratio 3.01, 95\% CI 0.91-9.91; $P=.07$ ).

\section{Discussion}

We enrolled 70 subjects who were admitted in the ICU and who required mechanical ventilation. The tracheostomy rate was on the high side of those reported in the literature, ${ }^{19}$ probably due to the severity of disease at ICU admission as assessed by the APACHE II score. ${ }^{20}$ Weaning outcomes depend on the balance between the ventilator demands and patient capacity. In this sense, respiratory muscle function is an essential factor for the ability to sustain spontaneous breathing without any assistance. ${ }^{21}$ The present study included a sample of subjects on prolonged weaning, with an average time of $17 \mathrm{~d}$ to the start of weaning, which implied that their respiratory muscles were already compromised to some extent. ${ }^{4,5,11}$

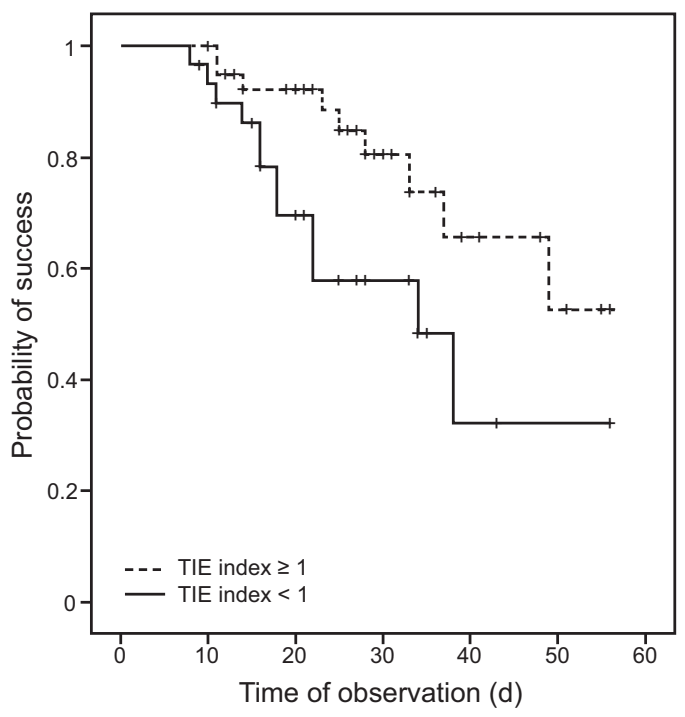

Fig. 4. Kaplan-Meier curves, showing the probability of weaning success in subjects with timed inspiratory effort (TIE) of $\geq 1 \mathrm{~cm}$ $\mathrm{H}_{2} \mathrm{O} / \mathrm{s}$ and $\mathrm{TIE}$ of $<1 \mathrm{~cm} \mathrm{H}_{2} \mathrm{O} / \mathrm{s}$ over a 56 -d period. $P=.030$ between groups.

Table 3. Cox Regression Analysis to Test for Association of the Timed Inspiratory Effort With Failure of Weaning Adjusted for Selected Variables

\begin{tabular}{lll}
\hline \hline \multicolumn{1}{c}{ Parameter } & $\begin{array}{c}\text { Hazard } \\
\text { Ratio (95\% CI) }\end{array}$ & $P$ \\
\hline Timed inspiratory effort $\geq 1 \mathrm{~cm} \mathrm{H}_{2} \mathrm{O} / \mathrm{s}$ & $0.36(0.15-0.91)$ & .030 \\
Age, y & $1.04(1.01-1.07)$ & .03 \\
Males & $0.52(0.25-1.10)$ & .09 \\
APACHE II score & $1.02(0.95-1.09)$ & .65 \\
Length to start weaning & $1.03(1.01-1.05)$ & .037 \\
Weaning duration & $1.00(0.97-1.03)$ & .91 \\
Interruptions of weaning trials & $3.01(0.91-9.91)$ & .07 \\
Alertness* & $0.67(0.30-1.50)$ & .33
\end{tabular}

*Yes if the Richmond Agitation-Sedation scale was from -1 to +1 . APACHE II = Acute Physiology and Chronic Health Evaluation II

To test if the timed inspiratory effort index would be useful to predict successful weaning in the study group, serial measurements of this parameter were performed from the beginning of the protocol until the end of the weaning process. The most common method to assess respiratory 
muscle function at the bedside is the $\mathrm{P}_{\text {Imax }}$. However, some studies have shown that it has low accuracy to predict weaning outcomes, even when using a unidirectional valve. ${ }^{6,13,14,22}$ Although a recent study found an accuracy of $0.80^{14,15}$ when using the unidirectional valve method, the use of the TIE index, which takes into account the time required to reach the $\mathrm{P}_{\text {Imax }}$ within a maximum observation period of $60 \mathrm{~s}$, provided an accuracy of 0.90 in a general sample of subjects in the ICU. ${ }^{14}$ Accordingly, we found an accuracy of 0.96, which confirmed the TIE index as a useful tool when assessing subjects with respiratory muscle dysfunction during the weaning process. ${ }^{6}$

In the present study, weaning failure was present in $37 \%$ of the subjects, and the ICU mortality was 50\%, $22(31 \%)$ of which occurred in subjects who had successful weaning. Our findings can be compared with 2 other studies. In the first study, the weaning failure rate was $47 \%$ and in-hospital mortality was $26 \% .^{5}$ In the second study, the weaning failure rate was $53 \%$ and ICU mortality was $36.8 \%$, with a hospital death rate of $58.6 \% .{ }^{23}$ The differences observed in our study may be explained, in part, by the advanced age of the subjects (median, $72 \mathrm{y}$ ), prolonged duration of mechanical ventilation (median, $17.5 \mathrm{~d}$ ), high prevalence of infection (58\%), and higher APACHE II scores.

The spontaneous breathing trial is the accepted standard to evaluate the success of weaning and extubation of patients on mechanical ventilation in the ICU. ${ }^{8,24,25}$ However, the failure rate of an spontaneous breathing trial to predict weaning success is $\sim 15 \%$, whereas the accuracy of the timed inspiratory effort index is $\sim 90 \% .{ }^{14}$ It is likely that the good performance shown by the timed inspiratory effort index stems is because its measurement considers the muscular response (time) in combination with the performance of the respiratory muscles. ${ }^{8,15}$ Essentially, a good screening test should include a low number of false-negative results, along with a high number of true-positive results. ${ }^{15,18}$ In our study, the sensitivity of the timed inspiratory effort index was $86.4 \%$ and the specificity was $96.2 \%$. These numbers translated the high reliability of the parameter to predict weaning failure or success.

Many predictive indexes were not incorporated into clinical practice because they presented low accuracy (area under the curve values receiver operating characteristic of $<0.80){ }^{14,15,18,26}$ In the present study, the accuracy of the TIE index, measured as the area under the receiver operating characteristic curve, was 0.93 , similar to previous studies which showed area under the curve values between 0.90 and $0.96 .6,14$ It is worth noting that the cutoff point found in the present study, which dealt with subjects with tracheostomy and in prolonged weaning ( $\geq 1.0 \mathrm{~cm} \mathrm{H} \mathrm{H}_{2} \mathrm{O} / \mathrm{s}$ ), matched the one reported for subjects with difficult weaning and with neuromuscular diseases. ${ }^{6}$

When the probability of success over a 56-d period was analyzed on a Kaplan-Meier curve factored by the cutoff value of the timed inspiratory effort index, a statistically significant difference was obtained in favor of values $\geq 1.0 \mathrm{~cm} \mathrm{H}_{2} \mathrm{O} / \mathrm{s}$. The finding confirmed our working hypothesis that the weekly serial measurement of the TIE index can help the decision to start the weaning process. In clinical practice, a TIE index of $\geq 1.0 \mathrm{~cm} \mathrm{H}_{2} \mathrm{O} / \mathrm{s}$ could be used as an indication that patients are ready to start the weaning process. Comparisons with previous studies could not be done because the use of the TIE index for this specific purpose is not reported in the literature.

In addition, we used a Cox regression multivariate model to test for the association of timed inspiratory effort index of $\geq 1.0 \mathrm{~cm} \mathrm{H}_{2} \mathrm{O} / \mathrm{s}$ with weaning failure. Even after adjustment for age, sex, APACHE II score, length of time to weaning initiation, weaning duration, weaning trial interruptions, and alertness, the risk of weaning failure was reduced by $64 \%$ when the TIE index value was $\geq 1.0 \mathrm{~cm} \mathrm{H}_{2} \mathrm{O} / \mathrm{s}$. Conversely, the risk rate increased with age, length of time to weaning initiation, and the number of weaning trial interruptions, but statistical significance was not found for the last variable.

Although alertness had no statistical significance in this model, it is considered a determining factor when using tools to measure lung function as well as to achieve ventilatory independence. ${ }^{15,27,28}$ In this context, patients with a low level of consciousness may present with a low $\mathrm{P}_{\text {Imax }} \cdot{ }^{27-29}$ However, the technique used for the assessment of the TIE index allows the progressive stimulation of the respiratory center with airway occlusion until no more increases in peak pressures are observed. ${ }^{13,14,27,28}$ Thus, the TIE index measurement does not require verbal stimulation by the examiner and can be performed in patients who are cooperative as well as in patients who are uncooperative. ${ }^{13,14}$ In this way, the absence of an association of the alertness with weaning failure in the present study cannot be seen as a surprise. Ventilatory independency mainly depends on an intact respiratory center combined with adequate respiratory muscle strength, variables well assessed during the measurement of the timed inspiratory effort index. ${ }^{13}$

Our study presented some limitations. The sample size of the present study was relatively small and derived from a sole center, which perhaps limited the generalizability of the findings. Although we had no objective information on the factors that led to a skeletal muscle injury in our sample, the timed inspiratory effort index was helpful to evaluate respiratory muscles function and to predict weaning outcome in subjects who were difficult to wean. Additional studies that confirm our results are needed before recommending such a strategy in clinical practice, and a randomized controlled trial would be needed to see if the timed inspiratory effort index leads to faster liberation from the ventilator. 


\section{Timed Inspiratory EfFort Index to Predict WeAning}

\section{Conclusions}

The weekly serial measurements of the timed inspiratory effort index proved to be efficient in predicting the success of weaning off mechanical ventilation in subjects with tracheostomy and classified as prolonged weaning.

\section{ACKNOWLEDGMENTS}

The authors thank the respiratory physiotherapists and physicians of the ICU of the Hospital Naval Marcílio Dias (Marinha do Brazil)

\section{REFERENCES}

1. Pham T, Brochard LJ, Slutsky AS. Mechanical Ventilation: State of the Art. Mayo Clin Proc 2017;92(9):1382-1400.

2. Ouellette DR, Patel S, Girard TD, Morris PE, Schmidt GA, Truwit $\mathrm{JD}$, et al. Liberation from mechanical ventilation in critically Ill adults: an official American College of Chest Physicians/American Thoracic Society Clinical Practice Guideline: Inspiratory Pressure Augmentation During Spontaneous Breathing Trials, Protocols Minimizing Sedation, and Noninvasive Ventilation Immediately After Extubation Chest 2017;151(1):166-180.

3. Levine S, Nguyen T, Taylor N, Friscia ME, Budak MT, Rothenberg $\mathrm{P}$, et al. Rapid disuse atrophy of diaphragm fibers in mechanically ventilated humans. N Engl J Med 2008;358(13):1327-1335.

4. Scheinhorn DJ, Hassenpflug MS, Votto JJ, Chao DC, Epstein SK, Doig GS, et al.; Ventilation Outcomes Study Group. Post-ICU mechanical ventilation at 23 long-term care hospitals: a multicenter outcomes study. Chest 2007;131(1):85-93.

5. Jubran A, Grant BJ, Duffner LA, Collins EG, Lanuza DM, Hoffman LA, Tobin MJ. Effect of pressure support vs unassisted breathing through a tracheostomy collar on weaning duration in patients requiring prolonged mechanical ventilation. A randomized trial. JAMA 2013;309(7):671-677.

6. de Souza LC, Guimarães FS, Lugon JR. The timed inspiratory effort: a promising index of mechanical ventilation weaning for patients with neurologic or neuromuscular diseases. Respir Care 2015;60(2): 231-238.

7. Bissett BM, Leditschke IA, Neeman T, Boots R, Paratz J. Inspiratory muscle training to enhance recovery from mechanical ventilation: a randomized trial. Thorax 2016;71(9):812-819.

8. Boles JM, Bion J, Connors A, Herridge M, Marsh B, Melot C, et al. Weaning from mechanical ventilation. Eur Respir J 2007;29(5): 1033 1056.

9. Béduneau G, Pham T, Schortgen F, Piquilloud L, Zogheib E, Jonas M, et al. WIND (Weaning according to a New Definition) Study Group and the REVA (Réseau Européen de Recherche en Ventilation Artificielle) Network. Epidemiology of Weaning Outcome according to a New Definition. The WIND Study. Am J Respir Crit Care Med 2017;195(6):772-783.

10. Stauffer JL, Fayter NA, Graves B, Cromb M, Lynch JC, Goebel P. Survival following mechanical ventilation for acute respiratory failure in adult men. Chest 1993;104(4):1222-1229.

11. Carson SS, Garrett J, Hanson LC, Lanier J, Govert J, Brake MC, et al. A prognostic model for one-year mortality in patients requiring prolonged mechanical ventilation. Crit Care Med 2008;36(7):20612069.

12. Nava S, Fasano L. Inspiratory muscle training in difficult to wean patients: work it harder, make it better, do it faster, makes us stronger. Crit Care 2011;15(2):153.
13. Leonardo Cordeiro de Souza. Timed inspiratory effort index, a new diagnostic method to assess endurance and inspiratory muscle strength in patients under mechanical ventilation. J Pulm Respir Med 2016; 6(6):1-4.

14. de Souza LC, Guimarães FS, Lugon JR. Evaluation of a new index of mechanical ventilation weaning: the timed inspiratory effort. J Intensive Care Med 2015;30(1):37-43.

15. de Souza LC, da Silva CT Jr, Almeida JR, Lugon JR. Comparison of maximal inspiratory pressure, tracheal airway occlusion pressure, and its ratio in the prediction of weaning outcome: impact of the use of a digital vacuometer and the unidirectional valve. Respir Care 2012;57(8):1285-1290.

16. Sessler CN, Gosnell MS, Grap MJ, Brophy GM, O’Neal PV, Keane $\mathrm{KA}$, et al. The Richmond Agitation-Sedation Scale: validity and reliability in adult intensive care patients. Am J Respir Crit Care Med 2002;166(10):1338-1344.

17. Nemer SN, Barbas CS, Caldeira JB, Cárias TC, Santos RG, Almeida $\mathrm{LC}$, et al. A new integrative weaning index of discontinuation from mechanical ventilation. Crit Care 2009;13(5):R152.

18. Yang KL, Tobin MJ. A prospective study of indexes predicting the outcome of trials of weaning from mechanical ventilation. N Engl J Med 1991;324(21):1445-1450.

19. Jeong BH, Ko MG, Nam J, Yoo H, Chung CR, Suh GY, Jeon K. Differences in clinical outcomes according to weaning classifications in medical intensive care units. PLoS One 2015;10(4):1-13.

20. Frost SA, Alexandrou E, Bogdanovski T, Salamonson Y, Davidson PM, Parr MJ, Hillman KM. Severity of illness and risk of readmission to intensive care: a meta-analysis. Resuscitation 2009;80(5): 505-510.

21. MacIntyre NR, Cook DJ, Ely EW Jr, Epstein SK, Fink JB, Heffner JE, et al.; American College of Chest Physicians; American Association for Respiratory Care; American College of Critical Care Medicine Evidence-based guidelines for weaning and discontinuing ventilatory support: a collective task force facilitated by the American College of Chest Physicians; the American Association for Respiratory Care; and the American College of Critical Care Medicine. Chest 2001;120(6 Suppl):375S-395S.

22. Caruso P, Albuquerque AL, Santana PV, Cardenas LZ, Ferreira JG, Prina E, et al. [Diagnostic methods to assess inspiratory and expiratory muscle strength]. J Bras Pneumol 2015;41(2):110-123.

23. Martin AD, Smith BK, Davenport P, Harman E, Gonzalez-Rothi RJ, $\mathrm{Baz} \mathrm{M}$ et al. Inspiratory muscle strength training improves weaning outcome in failure to wean patients: a randomized trial. Crit Care 2011;15(2):R84.

24. Epstein SK. Weaning from ventilatory support. Curr Opin Crit Care 2009;15(1):36-43.

25. Alam M, Jones G, Kahl W, Kamath MV. Modeling the weaning of intensive care unit patients from mechanical ventilation: a review. Crit Rev Biomed Eng 2014;42(1):25-61.

26. Barbas CS, Ísola AM, Farias AM, Cavalcanti AB, Gama AM, Duarte AC, et al. Brazilian recommendations of mechanical ventilation 2013. Part 2. Rev Bras Ter Intensiva 2014;26(3):215-239.

27. Guimarães FS, Alves FF, Constantino SS, Dias CM, Menezes SLS. Maximal inspiratory pressure evaluation among noncooperative critical patients: comparison between two methods. Rev Bras Fisioter 2007;11(11):233-238.

28. Marini JJ, Smith TC, Lamb V. Estimation of inspiratory muscle strength in mechanically ventilated patients: the measurement of maximal inspiratory pressure. J Crit Care Med 1986;1(1):32-38.

29. Soo Hoo GW, Park L. Variations in the measurement of weaning parameters: a survey of respiratory therapists. Chest 2002;121(6): 1947-1955. 